\title{
Delayed Brain Edema and Swelling following Craniectomy for Evacuation of an Epidural Abscess that Improved by Cranioplasty: Case Report
}

\author{
Narushi Sugii ${ }^{1}$ Masahide Matsuda ${ }^{1}$ Tomokazu Sekine ${ }^{1}$ Hideaki Matsumura ${ }^{1}$ Tetsuya Yamamoto ${ }^{1}$ \\ Akira Matsumura ${ }^{1}$ \\ ${ }^{1}$ Department of Neurosurgery, University of Tsukuba, Tsukuba, \\ Ibaraki, Japan \\ J Neurol Surg Rep 2017;78:e109-e112. \\ Address for correspondence Masahide Matsuda, MD, PhD, \\ Department of Neurosurgery, Faculty of Medicine, University of \\ Tsukuba, Tsukuba, Ibaraki 305-8575, Japan \\ (e-mail: m-matsuda@md.tsukuba.ac.jp).
}

\begin{abstract}
Keywords

- Brain Swelling

- bone flap removal

- delayed

- epidural abscess
\end{abstract}

\section{Introduction}

As a delayed complication following bone flap removal for subdural empyemas or epidural abscesses, sinking skin flap syndrome has been widely reported. After bone removal, the stretched scalp above the bone defect may sink due to the absence of underlying bone to support the atmospheric pressure. ${ }^{1,2}$ On the other hand, delayed brain swelling due to disruption in cerebrospinal fluid (CSF) circulation such as hydrocephalus or subdural effusion through the bone defect has also been frequently described. ${ }^{1,3,4}$ Surgical intervention such as ventriculoperitoneal shunting is required before bone flap replacement in some cases. We report a unique case of delayed brain swelling without CSF disruption following bone flap removal that improved rapidly after cranioplasty and discuss the potential mechanism underlying this delayed and reversible brain swelling.

\section{Case Report}

A 22-year-old woman without a notable previous medical history presented with intermittent progressive headache and nausea for 4 weeks. Magnetic resonance imaging (MRI) revealed a 5-cm extra-axial, gadolinium (Gd)-enhancing mass located in the left fronto-temporal convexity that was received

January 10, 2017

accepted after revision

June 5, 2017
DOI https://doi.org/

10.1055/s-0037-1606315. ISSN 2193-6358. (c) 2017 Georg Thieme Verlag KG
Stuttgart · New York

License terms

(c) $(1) \$$ 
associated with peritumoral brain edema ( - Fig. 1 A and B). She underwent a left fronto-temporal craniotomy for tumor removal. Although adhesion between the tumor and brain surface was strong, and the arachnoid membrane was partly disrupted, complete removal was achieved (Simpson grade I). Her dural defect was repaired with a free pericranial graft. The pathological diagnosis was meningothelial meningioma, the World Health Organization (WHO) grade I.

One month postoperatively, she developed purulent drainage from the incision sites. MRI showed epidural fluid collection around the surgical site (-Fig. 1C and D). She was taken to the operating room, and re-opening of the prior incision revealed purulent material in the epidural space. The abscess was evacuated, and the bone flap was removed. Vancomycin and ceftazidime were started as empiric therapy. Methicillin-resistant Staphylococcus aureus grew from a culture of the intraoperative specimen, and thus, the antibiotic regimen was changed to oral levofloxacin hydrate. Two weeks after the bone flap removal, her white blood cell count and C-reactive protein values returned to normal. She was discharged 1 month after the operation.

However, MRI, done 2 weeks after the bone flap removal, revealed brain edema around the defect in the skull, and the edematous brain showed focal swelling outward through the bone defect (-Fig. 2A). Despite the progression of brain edema and external swelling, her clinical course remained stable, and aggravation of laboratory parameters was not observed, even after the completion of 6 weeks of antimi-
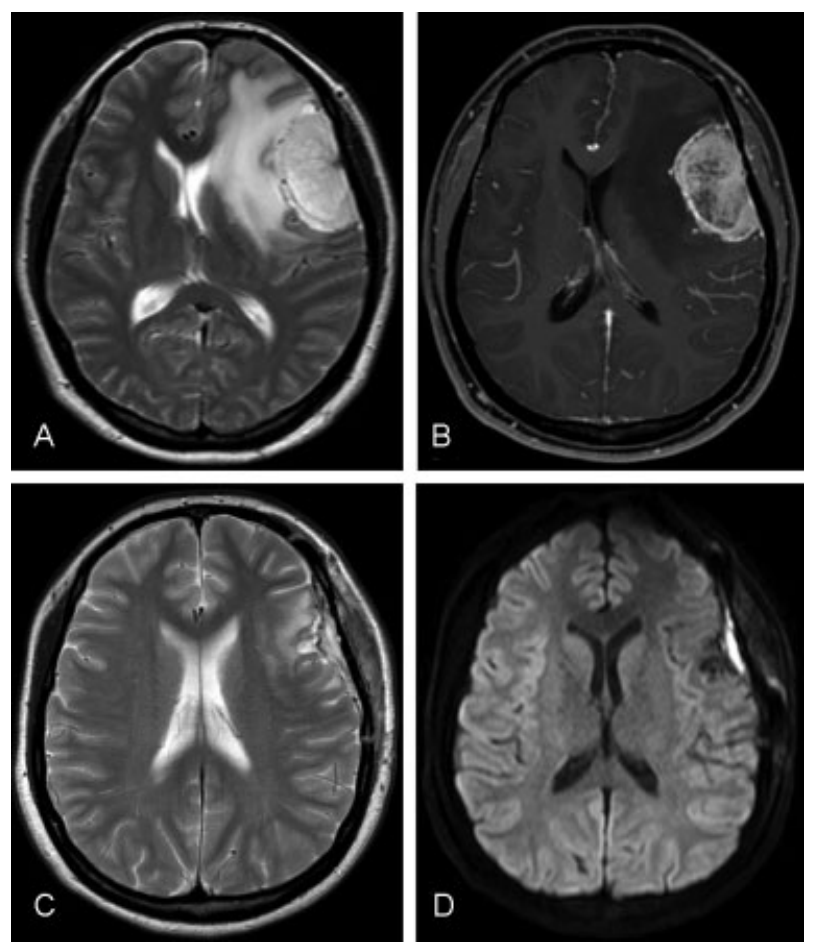

Fig. 1 Preoperative T2-weighted (A) and T1-weighted post-gadolinium contrast (B) axial magnetic resonance imaging showing an extraaxial mass with peritumoral edema in the adjacent brain parenchyma. T2-weighted (C) and diffusion-weighted (D) imaging 1 month after the first surgery, showing improvement in the peritumoral edema and the emergence of epidural fluid collection around the surgical site, indicating the epidural abscess. crobial therapy. We monitored her progress for another month without antibiotics. Although laboratory findings, including the erythrocyte sedimentation rate and C-reactive protein, showed no evidence of inflammation, worsening of the focal brain swelling without ventricle dilatation was observed on MRI (-Fig. 2B). We believed that the edema and swelling were caused by the very state of the bone defect, and thus, we decided to perform a cranioplasty 10 weeks after the bone flap removal. The dural autograft became thickened and adhered strongly to the musculocutaneous flap. Adhesion between the cerebral cortex and the dural substitute was also strong. Although the edematous brain gently swelled out intraoperatively, the brain could be easily adjusted to the correct position, indicating that the intracranial pressure seemed to remain only slightly elevated. The bone defect was covered with a synthetic bone graft.

MRI revealed amelioration of brain edema and swelling 1 week after cranioplasty ( $\mathbf{- F i g . ~} \mathbf{2 C}$ ), and the edema completely disappeared over the next 3 months (-Fig. 2D). She had no further postoperative complications or evidence of recurrent infection or meningioma over a follow-up period of $>3$ years.

\section{Discussion}

In the present case, despite resolution of postoperative surgical site infection (SSI), including an epidural abscess, brain edema and external swelling progressed gradually
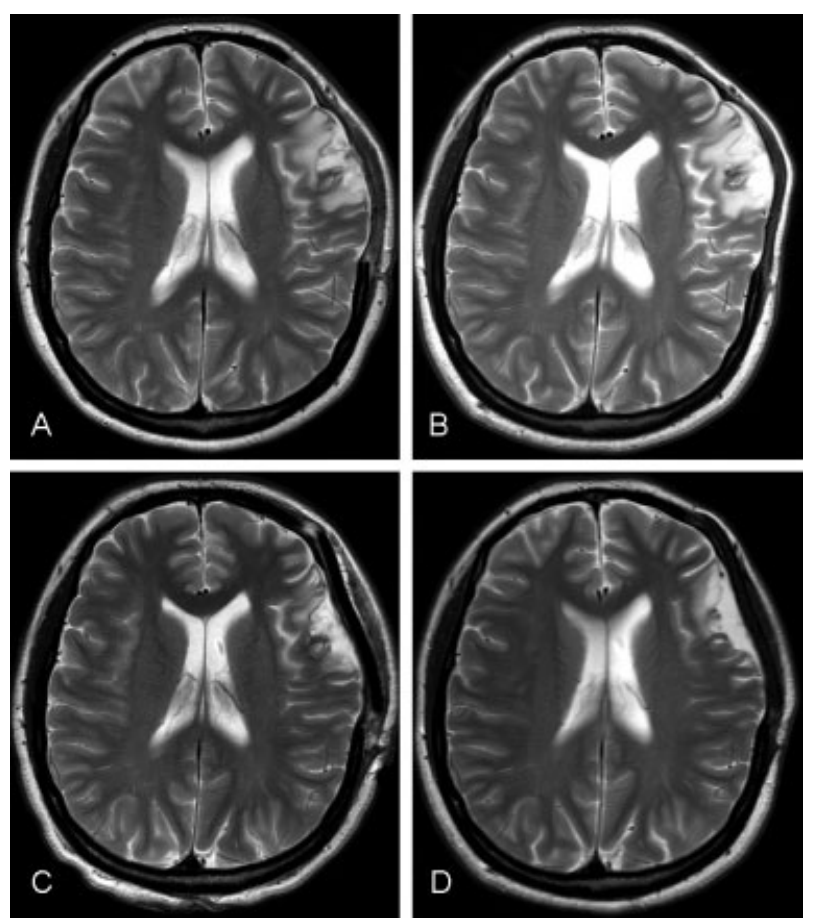

Fig. 2 T2-weighted axial imaging revealing the progression of focal brain edema and external swelling over 2 weeks (A) and 8 weeks (B) after the bone flap removal. T2-weighted imaging showing adjustment of the protruded brain into the correct position and amelioration of the edema 1 week after cranioplasty (C). Although a small amount of epidural effusion remained, the brain edema resolved completely 3 months after the bone flap removal (D). 
after the bone flap removal and were eventually improved by cranioplasty. The present case is unique in the following respect: localized brain edema and external swelling progressed slowly after the bone flap removal. In addition, even though the brain edema remained, it resolved soon after cranioplasty. To the best of our knowledge, no similar cases have been described.

Delayed brain swelling due to disruptions in CSF circulation such as hydrocephalus or subdural effusion is a common complication after a decompressive craniectomy and develops mainly following CSF malabsorption or obstructed CSF flow. ${ }^{1,3-5}$ The incidence is reported to be 0 to $88.2 \%$, depending on the various definitions in each study. ${ }^{3}$ Subdural effusion, when present, usually develops within 1 month after craniectomy, and hydrocephalus requiring a ventriculoperitoneal shunt, when present, generally occurs between 1 and 6 months. ${ }^{3,5,6}$ In the present case, because computed tomography/MRI revealed neither enlargement of ventricles nor subdural fluid collection before and after cranioplasty, the presence of CSF disruption was not suspected to be the cause of the delayed brain swelling.

Brain edema is mainly classified into vasogenic edema and cytotoxic edema. ${ }^{7}$ Vasogenic edema is more likely than cytotoxic edema to be the type of edema in the present case, considering the imaging findings and reversible clinical course. Any neurosurgical procedures may affect the structures surrounding the surgical site and may damage the vascular endothelium, leading to capillary leakage and blood-brain barrier disruption. ${ }^{8}$ Craniectomy, in particular, releases intracranial pressure outward through a defect in the skull and may lead to enlargement of extracellular spaces with consequent reduction in interstitial fluid pressure, finally promoting edema formation. ${ }^{9,10}$ Once the brain swells externally through the defect along with progression of brain edema, compression of cortical veins can be caused by shearing and pressure forces between the dural edge and brain tissue. ${ }^{11}$ Thus, venous congestion in the herniated part of the brain could be followed by a vicious cycle of brain edema.

Contrary to the self-limiting nature of the acute stage of edema, the fact that edema and external swelling progressed consistently beyond the subacute phase suggests the presence of another type of pathogenesis of edema, such as adhesion. The extent of surgical site adhesion between the inner surface of the dura and the pia-arachnoid membrane is directly affected by dural closure methods and the postoperative condition of the surgical field. ${ }^{12}$ In the present case, in addition to the usual formation of postsurgical adhesion, an inflammatory response induced by SSI may have enhanced the development of adhesion, and partial disruption of the pia-arachnoid membrane after meningioma removal may have predisposed the patient to the formation of cerebral-pia-arachnoid-dural adhesion. Also, we used a free pericranial flap as a dural substitute in the first surgery, and an autograft is more likely to develop adhesion compared with artificial dura mater. ${ }^{13}$ Under these circumstances, postsurgical adhesion among a cerebral cortex, pia, arachnoid, dura substitute, and musculocutaneous flap formed with kinked cortical veins or compression by the dural edge, leading to persistent brain edema and external swelling beyond the period of the acute phase. For brain edema that occurs according to this hypothesis, resolution of disruption of the cortical venous return is crucial for improving edema. In this case, kinking and compression of the cortical veins were resolved by adjusting the external protrusion of the cerebral cortex during cranioplasty, and consequently, brain edema improved.

No consensus exists regarding optimal timing for cranioplasty after SSI, but this procedure is usually performed in a delayed manner, once the infection is under control, and cerebral swelling has resolved. ${ }^{14-16}$ We adopted a careful wait-and-scan approach for 10 weeks after the bone flap removal. By this time, the brain edema did not improve, but worsened gradually on MRI. However, upon comprehensive consideration of the imaging findings, laboratory data, and her clinical course, we believed that pathological conditions, such as active infection, CSF disruptions, epileptic seizures, or severe elevation of intracranial pressure (ICP), were quite unlikely. Hence, we decided to perform the cranioplasty. Because the edematous brain could be easily returned to the correct position, and her postoperative course was uneventful, the decision to perform a cranioplasty at the time was considered reasonable and proper.

Our hypothesis about the underlying mechanism of delayed brain edema and swelling has several limitations. First, because we did not perform pre- and intra-operative vascular evaluation such as digital subtraction angiography, indocyanine green videoangiography, or a Doppler blood flow study, we could not confirm whether venous congestion was actually present or not. Second, a biopsy of the edematous brain was not performed. Therefore, we could not consider pathology that could be observed with this method. Third, brain edema and swelling undeniably improve as a natural course of the disease. Nevertheless, considering that the slowly progressive edema resolved soon after the cranioplasty, we believe that the edema improved due to the cranioplasty itself. Further case reports are warranted. In conclusion, we experienced a unique case of delayed brain swelling in the absence of CSF disruptions following bone flap removal that improved rapidly after cranioplasty. When focal brain edema and external swelling progress in a gradual and delayed manner after bone flap removal, after excluding other pathological conditions, such as increased ICP or exacerbation of infection, cranioplasty should be considered for improvement of cortical venous congestion caused by postsurgical adhesion.

\section{Conflict of Interest}

None.

\section{References}

1 Kurland DB, Khaladj-Ghom A, Stokum JA, et al. Complications associated with decompressive craniectomy: a systematic review. Neurocrit Care 2015;23(02):292-304. Doi: 10.1007/s12028-0150144-7

2 Sakamoto S, Eguchi K, Kiura Y, Arita K, Kurisu K. CT perfusion imaging in the syndrome of the sinking skin flap before and after 
e112 Delayed Brain Edema and Swelling Improved by Cranioplasty Sugii et al.

cranioplasty. Clin Neurol Neurosurg 2006;108(06):583-585. Doi: 10.1016/j.clineuro.2005.03.012

3 Ding J, Guo Y, Tian H. The influence of decompressive craniectomy on the development of hydrocephalus: a review. Arq Neuropsiquiatr 2014;72(09):715-720

4 Takeuchi S, Takasato Y, Masaoka H, et al. Hydrocephalus after decompressive craniectomy for hemispheric cerebral infarction. J Clin Neurosci 2013;20(03):377-382

5 Honeybul S, Ho KM. Decompressive craniectomy-a narrative review and discussion. Aust Crit Care 2014;27(02):85-91. Doi: 10.1016/j.aucc.2013.06.001

6 Yang XF, Wen L, Shen F, et al. Surgical complications secondary to decompressive craniectomy in patients with a head injury: a series of 108 consecutive cases. Acta Neurochir (Wien) 2008;150(12): 1241-1247, discussion 1248. Doi: 10.1007/s00701-008-0145-9

7 Michinaga S, Koyama Y. Pathogenesis of brain edema and investigation into anti-edema drugs. Int J Mol Sci 2015;16(05):9949-9975. Doi: 10.3390/ijms16059949

8 Sherchan P, Kim CH, Zhang JH. Surgical brain injury and edema prevention. Acta Neurochir Suppl (Wien) 2013;118:129-133. Doi: 10.1007/978-3-7091-1434-6_23

9 Cooper PR, Hagler H, Clark WK, Barnett P. Enhancement of experimental cerebral edema after decompressive craniectomy: implications for the management of severe head injuries. Neurosurgery 1979;4(04):296-300

10 Fung C, Murek M, Klinger-Gratz PP, et al. Effect of decompressive craniectomy on perihematomal edema in patients with intra- cerebral hemorrhage. PLoS One 2016;11(02):e0149169. Doi: 10.1371/journal.pone.0149169

11 Csókay A, Pataki G, Nagy L, Belán K. Vascular tunnel construction in the treatment of severe brain swelling caused by trauma and SAH. (evidence based on intra-operative blood flow measure). Neurol Res 2002;24(02):157-160. Doi: 10.1179/016164102101199701

12 Gonzalez-Lopez P, Harput MV, Türe H, Atalay B, Türe U. Efficacy of placing a thin layer of gelatin sponge over the subdural space during dural closure in preventing meningo-cerebral adhesion. World Neurosurg 2015;83(01):93-101. Doi: 10.1016/j.wneu.2014.02.032

13 Vakis A, Koutentakis D, Karabetsos D, Kalostos G. Use of polytetrafluoroethylene dural substitute as adhesion preventive material during craniectomies. Clin Neurol Neurosurg 2006;108(08): 798-802. Doi: 10.1016/j.clineuro.2005.11.026

14 Kshettry VR, Hardy S, Weil RJ, Angelov L, Barnett GH. Immediate titanium cranioplasty after debridement and craniectomy for postcraniotomy surgical site infection. Neurosurgery 2012; 70(1, Suppl Operative):8-14, discussion 14-15. Doi: 10.1227/ NEU.0b013e31822fef2c

15 Pierson M, Birinyi PV, Bhimireddy S, Coppens JR. Analysis of decompressive craniectomies with subsequent cranioplasties in the presence of collagen matrix dural substitute and polytetrafluoroethylene as an adhesion preventative material. World Neurosurg 2016;86:153-160. Doi: 10.1016/j.wneu.2015.09.078

16 Wind JJ, Ohaegbulam C, Iwamoto FM, Black PM, Park JK. Immediate titanium mesh cranioplasty for treatment of postcraniotomy infections. World Neurosurg 2013;79(01):207.e11-207.e13 